\title{
MINIMAL TIME PROBLEM WITH IMPULSIVE CONTROLS
}

\author{
KARL KUNISCH* AND ZHIPING RAO ${ }^{\dagger}$
}

\begin{abstract}
Time optimal control problems for systems with impulsive controls are investigated. Sufficient conditions for the existence of time optimal controls are given. A dynamical programming principle is derived and Lipschitz continuity of an appropriately defined value functional is established. The value functional satisfies a Hamilton-Jacobi-Bellman equation in the viscosity sense. A numerical example for a rider-swing system is presented and it is shown that the reachable set is enlargered by allowing for impulsive controls, when compared to nonimpulsive controls.
\end{abstract}

Key words. optimal control, impulsive differential equations, Hamilton-Jacobi-Bellman equations

AMS subject classifications. 49J15, 34A37, 34K35, 49Lxx

1 Introduction The present work aims at investigating the HamiltonJacobi-Bellman (HJB) approach for a minimal time problem with impulsive controls. The problem is based on the set of trajectories which are solutions to the following impulsive controlled dynamical system:

$$
\left\{\begin{array}{l}
\dot{y}(t)=f_{0}(y(t), u(t), \alpha(t))+\sum_{k=1}^{m} f_{k}(y(t), u(t), \alpha(t)) \dot{u}^{k}(t) \quad \text { for a.e. } t \in(0,+\infty), \\
y(0)=x, u(0)=u_{0},
\end{array}\right.
$$

where $\left(f_{k}\right)_{k=0, \ldots, m}$ are continuous functions, $y \in \mathbb{R}^{n}$ is the state variable and two inputs are involved in this controlled system: the input $u$ is a function of bounded variation (BV) taking values in a compact subset $U$ of $\mathbb{R}^{m}$ and the input $\alpha$ is a measurable function taking values in a compact subset $A$ of $\mathbb{R}^{p}$.

Given a closed target set $\mathcal{C} \subset \mathbb{R}^{n}$, for any initial data $\left(x, u_{0}\right) \in \mathbb{R}^{n} \times U$, consider the minimal time for the trajectories satisfying (1.1) to reach the target $\mathcal{C}$, i.e. let us introduce the function

$$
T\left(x, u_{0}\right):=\inf \left\{t: y\left(t^{+}\right) \in \mathcal{C}, y \text { satisfies }(1.1)\right\} .
$$

This definition of $T$ is still formal since the solutions of (1.1) are not yet properly defined. They will be defined rigorously in Section 3.

To motivate this study, let us consider the following example to show the potential of impulsive controls.

EXAMPLE 1. For the following system without impulsive controls:

$$
\dot{y}(t)=-y(t)+u(t),
$$

where the control $u$ takes value in $[-1,1]$, we have

$$
y(t)=y(0) e^{-t}+e^{-t} \int_{0}^{t} u(s) d s,
$$

\footnotetext{
*Institute for Mathematics and Scientific Computing, University of Graz, Heinrichstraße 36, A8010 Graz, Austria, and Radon Institute of Computational and Applied Mathematics, Austrian Academy of Sciences. Email: karl.kunisch@uni-graz.at

$\dagger(\otimes)$ Radon Institute of Computational and Applied Mathematics, Austrian Academy of Sciences, Altenbergerstraße 69, A-4040 Linz, Austria. Phone/fax: +43 (0)732 2468 5243/+43 (0)732 2468 5212. Email: zhiping.rao@ricam.oeaw.ac.at
} 
and the following estimate holds:

$$
|y(t)| \leq(|y(0)|+t) e^{-t} .
$$

If we take the target $\mathcal{C}=\{2\}$, then for the initial data $y(0)=1$, the target is not reachable.

Now if we replace the control $u$ by an impulsive control and consider

$$
\dot{y}(t)=-y(t)+\dot{u}(t)
$$

then

$$
y(t)=e^{-t} y(0)+u(t)-\int_{0}^{t} e^{s-t} u(s) d s
$$

In this case with the same target $\mathcal{C}=\{2\}$ and the same initial data $y(0)=1$, the target can be reached at finite time. For example, by taking $\tau>0, u:[0, \tau] \rightarrow \mathbb{R}$ such that

$$
u(t)=-1 \text { for } t \in[0, \tau) \text { and } u(\tau)=1
$$

the target is reached at $\tau$.

The dynamics of the system (1.1) depend not only on the value of the controls $u$ and $\alpha$, but also on the derivative of $u$. It is known that if $u$ is absolutely continuous, its derivative is integrable and therefore an absolutely continuous solution of (2.1) can be defined in the sense of Carathéodory. However, here $u$ is a BV function, which is discontinuous, and its derivative is interpreted as a distribution. According to the decomposition properties of BV functions, $u$ can only have countably many discontinuities of jump type ([1, Corollary 3.33]) and the derivative of $u$ gives an impulsive character to the dynamical system (1.1) whose solution has jump discontinuities as well. The main difficulty to define the solution of (1.1) lies in the fact that $f_{k}$ depends on $y$ and $u$ which implies that the magnitudes of the jumps of the trajectories are determined implicitly.

Impulsive dynamical systems arise in the modeling of problems in various domains such as Lagrangian mechanical system see [8, 17], neuroscience [10], resource management [13] and the reference therein. Several studies have been devoted to the definition of a solution concept for impulsive systems. The technique of graph completion was firstly used in [19] and formalized later in $[7,12,8]$ to define the jumps of the trajectories driven by the multiplication of the derivative of a discontinuous function with a state-dependent function. This graph completion concept has also been generalized to measure driven differential inclusions in [20,22]. Roughly speaking, a change of time scale $\mathcal{W}:[0,+\infty) \rightarrow[0,+\infty)$ is introduced such that at each moment $t$ of a jump of $u$, a fictive time interval is created in the new time scale. Subsequently an arc that connects the left and right limits of $u$ at $t$ is specified. This graph completion process leads to the following reparametrized system:

$$
\left\{\begin{aligned}
\dot{Z}(s) & =\sum_{k=0}^{m} F_{k}\left(Z(s), \alpha\left(\gamma_{0}(s)\right)\right) \dot{\gamma}_{k}(s), \text { a.e. } s \in(0,+\infty), \\
Z(0) & =\left(x, u_{0}\right)
\end{aligned}\right.
$$


where $Z(\cdot):=(y(\cdot), u(\cdot))$ and $\left(\gamma_{0}, \ldots, \gamma_{m}\right)(\cdot)$ is the graph completion of $u$. For this system, no jumps occur in the dynamics and $\left(\gamma_{0}, \ldots, \gamma_{m}\right)(\cdot)$ is absolutely continuous. Then the solution to the reparametrized system can be understood in the Carathéodory sense, and the trajectory $y$ of (1.1) is defined by the following equality:

$$
(y(\cdot), u(\cdot))=Z(\mathcal{W}(\cdot)) .
$$

We point out that the definition depends on the family of arcs that are chosen to complete the graph of $u$ in the new time scale, as studied in $[12,8]$. Different choices of graph completion may lead to different discontinuous trajectories. Only in some special cases (commutative cases), the definition is independent of this choice. In the present work, we consider only the graph completion with linear arcs, which are called canonical graph completions.

Once a precise definition of discontinuous trajectories is obtained, the minimal time problem can be rigorously defined. The impulse control problem has been widely studied, see [4,3] and the references therein. Optimality conditions for such problems have been studied in $[16,21]$ by applying the graph completion method, and recently in [2] with a more general class of impulsive controls. In [4, 3], the magnitudes of the jumps are considered as input controls, and this gives rise to quasi-variational inequalities which are analyzed by the theory of viscosity solutions. The problem with impulsive controls as the derivatives of absolutely continuous functions has been studied in [15] via a dynamical programming approach. The HJB approach for the minimal time problem that is studied in this paper has not been investigated in previous publications. Concerning the numerical results, this may be the first work which realizes impulsive controls. Earlier numerical results for impulsive systems with continuous controls can be found in $[9,18]$.

The minimal time function satisfies a classical Dynamic Programming Principle (DPP), but it is quite delicate to derive the HJB equation from the DPP due to the presence of the derivatives of $u$. Thanks to the reparametrization process, the original problem (1.2) can be turned into an equivalent control problem based on the reparametrized dynamical system: for any $x \in \mathbb{R}^{n}, u_{0} \in U$, consider

$$
S\left(x, u_{0}\right):=\inf \left\{s: Z(s) \in \mathcal{C} \times U, Z \text { satisfies }(1.3), Z(\cdot) \subset \mathbb{R}^{n} \times U\right\} .
$$

This is a standard minimal time problem with state constraints. Once this problem is solved with $u^{o p}$ an optimal control, the minimal time $T$ can be recovered by

$$
T\left(x, u_{0}\right)=\gamma_{0}^{o p}\left(S\left(x, u_{0}\right)\right),
$$

where $\gamma^{o p}$ is the canonical graph completion of $u^{o p}$.

Finally, to solve the reparametrized control problem (1.4) we consider a level set approach introduced in [5] for reachability problems under state constraints. This approach solves the problem by computing a HJB equation which does not rely on a controllability assumption.

The paper is organized as follows. The framework of problem is introduced in Section 2. Section 3 gives the precise definition of solutions of the impulsive systems based on the graph completions. In Section 4, the reparametrized control problem is discussed. Section 5 is devoted to the level set approach for the reparametrized control problem and the characterization of the backward reachable sets is obtained. An application on pumping of swing is given in Section 6 .

Notations. Throughout the paper, for any function $f:[0,+\infty) \rightarrow \mathbb{R}^{d}, f\left(t^{-}\right)$ stands for the left limit of $f$ at $t>0$ and $f\left(t^{+}\right)$stands for the right limit of $f$ at $t \geq 0$. 
2 Setting of the problem Let $U$ be a compact subset of $\mathbb{R}^{m}$ and $A$ be a compact subset of $\mathbb{R}^{p}$. For any $x \in \mathbb{R}^{n}, u_{0} \in U$, consider the following controlled dynamical system:

$$
\left\{\begin{array}{l}
\dot{y}(t)=f_{0}(y(t), u(t), \alpha(t))+\sum_{k=1}^{m} f_{k}(y(t), u(t), \alpha(t)) \dot{u}^{k}(t) \quad \text { for a.e. } t \in(0,+\infty), \\
y(0)=x, u(0)=u_{0} .
\end{array}\right.
$$

Here $y$ is the state variable, and $u=\left(u^{1}, \ldots, u^{k}\right)$ and $\alpha$ are the control variables. Given a constant $C_{1}>0$, let us define the sets

$$
\mathcal{A}:=L^{\infty}(0, \infty ; A)
$$

and

$$
\mathcal{U}:=\left\{u \in B V(0, \infty ; U): V_{0}^{\infty}\left(u^{k}\right) \leq C_{1}, k=1, \ldots, m\right\},
$$

where $B V(0,+\infty ; U)$ denotes the set of functions from $[0,+\infty)$ into $U$ with bounded total variations, and $V_{0}^{\infty}\left(u^{k}\right)$ denotes the total variation of the function $u^{k}$ on the interval $[0,+\infty)$.

For $i=0, \ldots, m$, we make the following assumptions for $f_{i}$ :

(H1) $f_{i}: \mathbb{R}^{n} \times U \times A \rightarrow \mathbb{R}^{n}$ is continuous in all variables and bounded by a positive constant $M$. Moreover, there exists a constant $L>0$ such that

$$
\left|f_{i}(x, u, a)-f_{i}(z, u, a)\right| \leq L|x-z|, \forall x, z \in \mathbb{R}^{n}, u \in U, a \in A .
$$

3 Impulsive control system At first the concept of solution to the impulsive control system (2.1) is specified. The idea is to add an extra state variable $z \in \mathbb{R}^{m}$ and the additional equation

$$
\dot{z}(t)=\dot{u}(t) \text { for a.e. } t \in(0,+\infty) .
$$

Then by introducing the variable $Y=\left(\begin{array}{c}y \\ z\end{array}\right)$ and the initial data $X=\left(\begin{array}{c}x \\ u_{0}\end{array}\right)$, the system (2.1) is turned into

$$
\left\{\begin{array}{l}
\dot{Y}(t)=\left(\begin{array}{l}
\dot{y}(t) \\
\dot{z}(t)
\end{array}\right)=\left(\begin{array}{c}
f_{0}(Y(t), \alpha(t)) \\
0
\end{array}\right)+\sum_{k=1}^{m}\left(\begin{array}{c}
f_{k}(Y(t), \alpha(t)) \\
e_{k}
\end{array}\right) \dot{u}^{k}(t), \\
Y(0)=X
\end{array}\right.
$$

where $\left(e_{k}\right)_{k=1, \ldots, m}$ is the Euclidean basis of $\mathbb{R}^{m}$.

Following [6, 12], the definition of solutions to (3.1) is based on the concept of graph completion. For any $u \in \mathcal{U}$, let $\mathcal{T}$ be the set of all the discontinuity points of $u$ which is at most countable. We define

$$
\mathcal{W}(t):=t+\sum_{k=1}^{m} V_{0}^{t}\left(u^{k}\right), \text { for } t \in[0,+\infty)
$$

where $V_{0}^{t}\left(u^{k}\right)$ denotes the total variation of $u^{k}$ on $[0, t]$ which is right continuous. Then $\mathcal{W}$ is strictly increasing and continuous on $[0,+\infty) \backslash \mathcal{T}$. We define the canonical graph completion of $u$ as a continuous path $\gamma=\left(\gamma_{0} ; \gamma_{1}, \ldots, \gamma_{m}\right):[0,+\infty) \rightarrow[0, \infty) \times \mathbb{R}^{m}$ such that 
- for $s=\mathcal{W}\left(t^{-}\right)$with $t \in[0,+\infty) \backslash \mathcal{T}$,

$$
\gamma(s)=(t ; u(t))
$$

- for $s \in\left[\mathcal{W}\left(t^{-}\right), \mathcal{W}\left(t^{+}\right)\right], t \in \mathcal{T}$

$$
\gamma(s)=\left(t ; u\left(t^{-}\right)+\frac{u\left(t^{+}\right)-u\left(t^{-}\right)}{\mathcal{W}\left(t^{+}\right)-\mathcal{W}\left(t^{-}\right)}\left(s-\mathcal{W}\left(t^{-}\right)\right)\right) .
$$

The path $\gamma$ leads to a reparametrization of the graph of $u$, and to the following reparametrized dynamical system

$$
\left\{\begin{array}{l}
\dot{Z}(s)=F_{0}\left(Z(s), \alpha\left(\gamma_{0}(s)\right)\right) \dot{\gamma}_{0}(s)+\sum_{k=1}^{m} F_{k}\left(Z(s), \alpha\left(\gamma_{0}(s)\right)\right) \dot{\gamma}_{k}(s), \text { a.e. } s \in(0,+\infty), \\
Z(0)=X
\end{array}\right.
$$

which has a unique absolutely continuous Carathéodory solution.

Now we are prepared to give the definition of the solution to (3.1).

Definition 3.1. For given $u \in \mathcal{U}, \alpha \in \mathcal{A}$, the solution to (3.1) is defined as

$$
Y(t):=Z(\mathcal{W}(t)), \forall t \in[0,+\infty),
$$

where $Z$ is the solution to (3.3) and $\mathcal{W}$ is defined as in (3.2).

Remark 3.2. We mention that any $B V$ function $u \in \mathcal{U}$ can be decomposed as $u=u_{a c}+u_{j}+u_{c}$ where $u_{a c}$ is absolutely continuous, $u_{j}$ is a jump function and $u_{c}$ is a cantor function (see [1] for more details). The graph completion technique turns the jump part $u_{j}$ and the cantor part $u_{c}$ into absolutely continuous functions.

Given a closed target set $\mathcal{C} \subset \mathbb{R}^{n}$, the minimal time function $T$ can now be defined as follows: for any $X \in \mathbb{R}^{n} \times U$,

$$
T(X)=\inf \left\{t: Y\left(t^{+}\right) \in \mathcal{C} \times U,(Y, u, \alpha) \text { satisfies }(3.1), u \in \mathcal{U}, \alpha \in \mathcal{A}\right\} .
$$

REMARK 3.3. Remark that if $u$ is right continuous then $\mathcal{W}$ is right continuous, which implies that $Y$ is right continuous. We point out that for any $u \in \mathcal{U}, u$ can be replaced by its right continuous good representative without changing the definition of the minimal time function T. See [1] for more details on the good representatives of $B V$ functions.

The following result concerns the existence of optimal controls.

TheOREM 3.4. Assume (H1). If $F_{k}, k=0, \ldots, m$ are independent of a, then for any $X \in \mathbb{R}^{n} \times U$ such that $T(X)<+\infty$ the minimal time problem (3.4) has an optimal solution. To prove this theorem, we need the following result ([12, Theorem $4.2])$.

LEMma 3.5. Under the assumptions of Theorem 3.4, let $\left(u_{n}\right)$ be a sequence in $\mathcal{U}$ and let $u \in \mathcal{U}$. Assume that for some $T>0$,

(i) $\lim _{n \rightarrow \infty} u_{n}(t)=u(t)$, for a.e. $t \in[0, T]$;

(ii) $\lim _{n \rightarrow \infty} V_{0}^{T}\left(u_{n}^{k}\right)=V_{0}^{T}\left(u^{k}\right), \forall k=1, \ldots, m$.

Let $Y_{n}$ and $Y$ be the solutions to (3.1) on $[0, T]$ corresponding to the controls $u_{n}$ and $u$ respectively. Then

$$
\lim _{n \rightarrow+\infty} Y_{n}(t)=Y(t)
$$

for each $t \in[0, T]$ where $u$ is continuous. The proof of theorem 3.4 is given next. 
Proof. For any $X \in \mathbb{R}^{n} \times U$ with $T(X)<+\infty$, there exist $u_{n} \in \mathcal{U}, t_{n} \geq 0$ such that $\left(Y_{n}, u_{n}\right)$ satisfies (3.1) with

$$
Y_{n}\left(t_{n}\right) \in \mathcal{C} \times U, t_{n} \rightarrow T(X) .
$$

For any $\tilde{T}>T(X)$, we have $u_{n} \in B V(0, \tilde{T} ; U)$. Since $u_{n}$ is bounded in $B V(0, \tilde{T} ; U)$, using the fact that the embedding $B V(0, \tilde{T} ; U) \hookrightarrow L^{p}(0, \tilde{T} ; U)$ is compact for $1 \leq p<$ $\infty$ ([1, Corollary 3.49]), there exists $u \in L^{1}(0, \tilde{T} ; U)$ such that

$$
u_{n} \rightarrow u \text { in } L^{1}(0, \tilde{T} ; U),
$$

up to a subsequence. Then we obtain that

$$
u_{n} \rightarrow u \text { a.e. in }[0, \tilde{T}] .
$$

Moreover, since $B V(0, \tilde{T} ; U)$ is a Banach space and a subspace of $L^{1}(0, \tilde{T} ; U)$, we deduce that $u_{n} \rightarrow u$ in $B V(0, \tilde{T} ; U)$. Thus,

$$
\lim _{n \rightarrow \infty} V_{0}^{T}\left(u_{n}^{k}\right)=V_{0}^{T}\left(u^{k}\right), \forall k=1, \ldots, m .
$$

Let $Y$ be the solution to (3.1) corresponding to the control $u$, then by lemma 3.5

$$
\lim _{n \rightarrow+\infty} Y_{n}(t)=Y(t)
$$

for each $t \in[0, \tilde{T}]$ where $u$ is continuous.

Due to remark 3.3, without loss of generality, suppose that $u_{n}$ and $u$ are right continuous. Then $Y_{n}$ and $Y$ are right continuous. We construct the new trajectories $\tilde{Y}_{n}$ as follows:

$$
\tilde{Y}_{n}(t)=Y_{n}(t), \text { for } 0 \leq t \leq t_{n}
$$

and $\tilde{Y}_{n}$ satisfies

$$
\dot{\tilde{Y}}_{n}(t)=f_{0}\left(\tilde{Y}_{n}(t), u_{n}\left(t_{n}\right)\right) \text {, for } t>t_{n} .
$$

Since $f_{0}$ is bounded by $M$, we have

$$
\left|\tilde{Y}_{n}(t)-\tilde{Y}_{n}\left(t_{n}\right)\right| \leq M\left|t-t_{n}\right|, \text { for } t \geq t_{n} .
$$

Let $\tau>T(X)$ such that $Y$ is continuous at $\tau$ and

$$
|\tau-T(X)|<\varepsilon,|Y(\tau)-Y(T(X))|<\varepsilon .
$$

Since $\tilde{Y}_{n}(\tau) \rightarrow Y(\tau)$, then let $n$ be big enough such that

$$
\left|\tilde{Y}_{n}(\tau)-Y(\tau)\right|<\varepsilon,\left|t_{n}-T(X)\right|<\varepsilon, t_{n}<\tau .
$$

Finally we have

$$
\begin{aligned}
\left|\tilde{Y}_{n}\left(t_{n}\right)-Y(T(X))\right| & \leq\left|\tilde{Y}_{n}\left(t_{n}\right)-\tilde{Y}_{n}(\tau)\right|+\left|\tilde{Y}_{n}(\tau)-Y(\tau)\right|+|Y(\tau)-Y(T(X))| \\
& \leq 2(M+1) \varepsilon,
\end{aligned}
$$

for any $\varepsilon>0$. Then we deduce that $Y(T(X)) \in \mathcal{C}$ since $\mathcal{C}$ is closed.

The above result guarantees existence of optimal controls if the control system is independent of $\alpha$. In case the dynamics depend on $\alpha$ one has to cope with lack convexity due to the nonlinearity of $f_{k}, k=0, \ldots, m$. In the following sections, we nevertheless allow the appearance of $\alpha$ for the derivation of the HJB theory. 
4 Reparametrized control problem Let $\Gamma$ be the set of all the graph completions:

$$
\Gamma=\{\gamma:[0, \infty) \rightarrow[0, \infty) \times U: \gamma \text { is the canonical graph completion of } u, u \in \mathcal{U}\}
$$

We define the set of state constraints $\mathcal{K}=\mathbb{R}^{n} \times U$ and the set of admissible trajectories

$$
\Phi[X]:=\{Z:[0,+\infty) \rightarrow \mathcal{K},(Z, \gamma, \alpha) \text { satisfies }(3.3), \gamma \in \Gamma, \alpha \in \mathcal{A}\} .
$$

The reparametrized control problem is the following: given $X \in \mathcal{K}$,

$$
S(X):=\inf \{s: Z(s) \in \mathcal{C}, Z(\cdot) \in \Phi[X]\} .
$$

We now discuss the relation between the original problem and the reparametrized problem.

TheOREM 4.1. Assume (H1) and assume in addition that $U$ is convex. Then for any $X \in \mathcal{K}$, one of the following holds.

- $T(X)=S(X)=+\infty$.

- If $T(X)<+\infty$ and $u^{o p} \in \mathcal{U}$ and $\alpha^{o p} \in \mathcal{A}$ are optimal controls for problem (3.4) at $X$, then one has

$$
T(X)=\gamma_{0}^{o p}(S(X))
$$

where $\gamma^{o p}$ is the corresponding graph completion of $u^{o p}$.

Proof. For any $X \in \mathcal{K}$, we consider two cases: $T(X)=+\infty$ and $T(X)<+\infty$.

Case 1: $T(X)=+\infty$.

If $S(X) \neq+\infty$, then for any $\varepsilon>0$, there exists some $Z^{\varepsilon} \in \Phi[X]$ with the associated control $\alpha^{\varepsilon} \in \mathcal{A}, \gamma^{\varepsilon} \in \Gamma$ such that

$$
Z^{\varepsilon}(S(X)+\varepsilon) \in \mathcal{C} .
$$

Let $u^{\varepsilon} \in \mathcal{U}$ and let $\gamma^{\varepsilon}$ be the graph completion of $u^{\varepsilon}$. Then $Y^{\varepsilon}(\cdot)=Z^{\varepsilon}(\mathcal{W}(\cdot))$ satisfies (3.1) with the control $u^{\varepsilon}$ and $\alpha^{\varepsilon}$ and

$$
Y^{\varepsilon}\left(\gamma_{0}^{\varepsilon}(S(X)+\varepsilon)\right) \in \mathcal{C} .
$$

Thus, $T(X) \leq \gamma_{0}^{\varepsilon}(S(X)+\varepsilon)<+\infty$ which is a contradiction. We then deduce that

$$
S(X)=+\infty .
$$

Case 2: $T(X)<+\infty$.

Let $y^{o p}$ be the optimal trajectory satisfying (2.1) associated with the controls $u^{o p}$ and $\alpha^{o p}, Y^{o p}:=\left(y^{o p}, u^{o p}\right)$ and $Z^{o p}$ be the solution to (3.3) associated with $\gamma^{o p}$ and $\alpha^{o p}$. Then it holds

$$
Z^{o p}\left(\mathcal{W}\left(t^{-}\right)\right)=Y^{o p}(t), \forall t \in[0,+\infty) .
$$

Moreover, since $u^{o p}(t) \in U$ for all $t \in[0,+\infty)$ and $U$ is convex, it follows that

$$
\gamma_{k}^{o p}(s) \in U, \forall s \in[0,+\infty) .
$$


We then deduce that

$$
Z^{o p}(s) \in \mathcal{K}, \forall s \in[0,+\infty)
$$

Thus,

$$
S(X) \leq \mathcal{W}\left(T(X)^{-}\right)
$$

i.e.

$$
\gamma_{0}^{o p}(S(X)) \leq T(X) .
$$

If $\gamma_{0}^{o p}(S(X))<T(X)$, let $\tilde{Z}$ be the optimal trajectory for the problem (4.1) at $X$ with the controls $\tilde{\gamma}$ and $\tilde{\alpha}$, then $(\tilde{y}(\cdot), \tilde{u}(\cdot)):=\tilde{Z}\left(\mathcal{W}\left(\cdot \cdot^{-}\right)\right)$satisfies $(2.1)$ and $\tilde{y}$ reaches the target $\mathcal{C}$ at time $\gamma_{0}^{o p}(S(X))$. It is a contradiction to the definition of $T(X)$. We then conclude that

$$
\gamma_{0}^{o p}(S(X))=T(X) .
$$

$\square$ Thanks to the above result, the problem of computing $T$ turns into the problem of computing $S$.

5 Characterization of the capture basin In the absence of controllability conditions, $S$ can be discontinuous on some regions which are not reachable for any of the admissible trajectories. To get the characterization result of $S$, some extra conditions are needed on the boundary of the reachable sets. In [5], a level set approach has been studied for the minimal time problem without jumps in the controls which leads to a characterization of the backward reachable sets. We shall follow this idea to transfer the problem (4.1) into the characterization of the capture basin of the target.

For any $s \geq 0$, let us define the capture basin of the target $\mathcal{C}$ at time $s$ :

$$
\operatorname{Cap}_{\mathcal{C}}(s):=\left\{X \in \mathbb{R}^{n+m}: \exists Z(\cdot) \in \Phi[X] \text { such that } Z(s) \in \mathcal{C}\right\} .
$$

Consider a Lipschitz continuous function $\varphi: \mathbb{R}^{n+m} \rightarrow \mathbb{R}$ satisfying

$$
X \in \mathcal{C} \Leftrightarrow \varphi(X) \leq 0 .
$$

The level set approach leads to introduce the following function: for any $s \in[0,+\infty)$, $X \in \mathbb{R}^{n+m}$,

$$
v(s, X):=\inf \{\varphi(Z(s)): Z(\cdot) \in \Phi[X]\},
$$

with the convention $\inf \emptyset=+\infty$. Then the capture basin $\operatorname{Cap}_{\mathcal{C}}(\cdot)$ is characterized by

$$
\operatorname{Cap}_{\mathcal{C}}(s)=\{X: v(s, X) \leq 0\} \text {, for } s \geq 0 .
$$

Note that $v$ is the value function of a Mayer's optimal control problem, and hence it can be characterized by a Hamilton-Jacobi-Bellman equation. But in presence of state constraints and in the absence of controllability conditions, it is not evident to characterize $v$ directly. Following $[5,14]$, we take a Lipschitz continuous function $g: \mathbb{R}^{n+m} \rightarrow \mathbb{R}$ such that

$$
g(X) \leq 0 \Leftrightarrow X \in \mathcal{K}, \text { where } X \in \mathbb{R}^{n+m} .
$$


The function $g$ can be taken as the signed distance function for example. Consider the new control problem

$$
w(s, X):=\inf _{\gamma \in \Gamma, \alpha \in \mathcal{A}}\left\{\max \left(\varphi(Z(s)), \max _{\theta \in[0, s]} g(Z(\theta))\right):(Z, \gamma, \alpha) \text { satisfies (3.3) }\right\},
$$

where compared to (5.1) the constraints on the control are made explicit.

Then we have the following result.

TheOREm 5.1. For any $s \geq 0$, the capture basin is given by

$$
\operatorname{Cap}_{\mathcal{C}}(s)=\{X: v(s, X) \leq 0\}=\{X: w(s, X) \leq 0\} .
$$

Further,

$$
S_{*}(X)=\inf \{s \geq 0: w(s, X) \leq 0\},
$$

where $S_{*}$ is the lower semi-continuous envelop of $S$.

Proof. We refer to [5] for the proof of (5.2).

Turning to the verification of (5.3), let $X \in \mathbb{R}^{d}$, we set $\tilde{s}=\inf \{s \geq 0: w(s, X) \leq$ $0\}$. If $\tilde{s}=+\infty$, then $S_{*}(X) \leq \tilde{s}$. Otherwise, if $\tilde{s}<+\infty$, the continuity of $w$ implies that for any $\varepsilon>0$ there exists $\varepsilon^{\prime} \in[0, \varepsilon]$ such that

$$
w\left(\tilde{s}+\varepsilon^{\prime}, X\right) \leq 0 .
$$

Thus, $X \in \operatorname{Cap}_{\mathcal{C}}\left(\tilde{s}+\varepsilon^{\prime}\right)$ which implies that

$$
S(X) \leq \tilde{s}+\varepsilon^{\prime} \leq \tilde{s}+\varepsilon .
$$

The arbitrary choice of $\varepsilon$ implies that $S(X) \leq \tilde{s}$. We then deduce that $S_{*}(X) \leq \tilde{s}$.

We proceed to prove that $\tilde{s} \leq S_{*}(X)$. Without loss of generality, suppose that $S_{*}(X)<+\infty$. Let $X_{n} \in \mathbb{R}^{d}, X_{n} \rightarrow X$ such that

$$
S_{*}(X)=\lim _{X_{n} \rightarrow X} S\left(X_{n}\right), S\left(X_{n}\right)<+\infty .
$$

For any $\varepsilon>0$, there exists $\varepsilon_{n} \in[0, \varepsilon]$ such that $X_{n} \in \operatorname{Cap}_{\mathcal{C}}\left(S\left(X_{n}\right)+\varepsilon_{n}\right)$, i.e.

$$
w\left(S\left(X_{n}\right)+\varepsilon_{n}, X_{n}\right) \leq 0 .
$$

Using the fact that $\varepsilon_{n} \rightarrow \varepsilon^{\prime}$ up to a subsequence where $\varepsilon^{\prime} \in[0, \varepsilon]$ and $w$ is continuous, we obtain

$$
w\left(S_{*}(X)+\varepsilon^{\prime}, X\right) \leq 0,
$$

which implies that

$$
\tilde{s} \leq S_{*}(X)+\varepsilon^{\prime} \leq S_{*}(X)+\varepsilon .
$$

The arbitrary choice of $\varepsilon$ then implies that $\tilde{s} \leq S_{*}(X)$.

Finally, we conclude that $S_{*}(X)=\tilde{s}$.

REMARK 5.2. In general, the minimal time function $S$ is not lower semi-continuous. However, if the set of dynamics is convex everywhere, then lower semi-continuity is guaranteed. 
Remark 5.3. The continuity of $w$, which is proved later in Proposition 5.7, is used in the above proof of Theorem 5.1. We point out that the proof of Proposition 5.7 is independent of Theorem 5.1.

In (3.3), it suffices that $\gamma$ is almost everywhere differentiable for the control system to be well defined. However, in the Hamiltonian which will be introduced later we need that $\dot{\gamma}$ is well defined everywhere. We set

$$
\Theta:=\left\{\lim \left(\dot{\gamma}_{0}\left(s_{n}\right), \ldots, \dot{\gamma}_{m}\left(s_{n}\right)\right): s_{n} \rightarrow s, s \in(0,+\infty), s_{n} \notin \Omega_{\gamma}\right\},
$$

where $\Omega_{\gamma}$ is the set of points in $(0,+\infty)$ at which $\gamma$ fails to be differentiable. The closed convex hull of $\Theta$ follows the concept of Clark's derivation.

Proposition 5.4. $\Theta=\left\{\left(\eta_{0}, \ldots, \eta_{m}\right) \in \mathbb{R}^{m+1}: \eta_{0} \geq 0, \sum_{k=0}^{m}\left|\eta_{k}\right|=1\right\}$.

Proof. Let us set $\Psi:=\left\{\left(\eta_{0}, \ldots, \eta_{m}\right) \in \mathbb{R}^{m+1}: \eta_{0} \geq 0, \sum_{k=0}^{m}\left|\eta_{k}\right|=1\right\}$.

At first, we prove that $\Theta \subset \Psi$. For any $u \in \mathcal{U}$, let $\gamma \in \Gamma$ be the graph completion of $u$. For each $s \in \Omega_{\gamma}$, if $s=\mathcal{W}(t)$ for some $t \in(0,+\infty)$, it holds that

$$
\dot{\gamma}_{0}(s)=\frac{1}{\dot{\mathcal{W}}(t)}=\frac{1}{1+\sum_{k=1}^{m}\left|\dot{u}^{k}(t)\right|}, \dot{\gamma}_{k}(s)=\dot{\gamma}_{0}(s) \dot{u}^{k}(t), \text { for } k=1, \ldots, m,
$$

which implies that

$$
0<\dot{\gamma}_{0}(s) \leq 1, \sum_{k=0}^{m}\left|\dot{\gamma}_{k}\right|=1
$$

If, on the other hand, $s \in\left(\mathcal{W}\left(t^{-}\right), \mathcal{W}\left(t^{+}\right)\right)$for some $t \in(0,+\infty)$ where $u$ jumps at time $t$, we have

$$
\dot{\gamma}_{0}(s)=0,\left|\dot{\gamma}_{k}(s)\right|=\frac{\left|u^{k}\left(t^{+}\right)-u^{k}\left(t^{-}\right)\right|}{\sum_{i=1}^{m}\left|V_{0}^{t^{+}}\left(u^{i}\right)-V_{0}^{t^{-}}\left(u^{i}\right)\right|} \leq 1, \text { for } k=1, \ldots, m,
$$

which implies that

$$
\sum_{k=0}^{m}\left|\dot{\gamma}_{k}\right|=1
$$

Finally, by the definition of $\Theta$ and the density of $\Omega_{\gamma}$ in $[0, \infty)$, we conclude that $\Theta \subset \Psi$.

We proceed to prove that $\Psi \subset \Theta$. Given any $\left(\eta_{0}, \ldots, \eta_{m}\right) \in \Psi$, we claim that there exists $u \in \mathcal{U}$ such that its graph completion $\gamma$ satisfies $\dot{\gamma}_{k}\left(s_{0}\right)=\eta_{k}, k=0, \ldots, m$ for some $s_{0}>0$. If $\eta_{0}>0$, let $\delta>0, t_{0}>\delta$ and

$$
u^{k}(t):= \begin{cases}\frac{\eta_{k}}{\eta_{0}} t & \text { if } t \in\left[t_{0}-\delta, t_{0}+\delta\right] \\ 0 & \text { otherwise }\end{cases}
$$

for $k=1, \ldots, m$. Then $u=\left(u^{1}, \ldots, u^{k}\right)$ satisfies

$$
\dot{\gamma}_{0}\left(s_{0}\right)=\frac{1}{1+\sum_{k=1}^{m}\left|\dot{u}^{k}\left(t_{0}\right)\right|}=\eta_{0}, \dot{\gamma}_{k}\left(s_{0}\right)=\dot{\gamma}_{0}\left(s_{0}\right) \dot{u}^{k}\left(t_{0}\right)=\gamma_{k}, \text { for } k=1, \ldots, m,
$$

where $s_{0}=\mathcal{W}\left(t_{0}\right)$. 
Otherwise $\eta_{0}=0$. Let $t_{0}>0$ and

$$
u^{k}(t):= \begin{cases}\eta_{k} & \text { for } t \geq t_{0} \\ 0 & \text { for } t<t_{0}\end{cases}
$$

for $k=1, \ldots, m$. Then for any $s_{0} \in\left(\mathcal{W}\left(t_{0}^{-}\right), \mathcal{W}\left(t_{0}^{+}\right)\right), u=\left(u^{1}, \ldots, u^{k}\right)$ satisfies

$$
\dot{\gamma}_{0}\left(s_{0}\right)=0, \dot{\gamma}_{k}\left(s_{0}\right)=\frac{\eta_{k}}{\sum_{i=1}^{m}\left|\eta_{i}\right|}=\eta_{k}, \text { for } k=1, \ldots, m .
$$

It is then deduced that $\Psi \subset \Theta$, which ends the proof. $\square$

We introduce the Hamiltonian $H: \mathbb{R}^{n+m} \times \mathbb{R}^{n+m} \rightarrow \mathbb{R}$

$$
H(X, p):=\sup _{a \in A, \eta \in \Theta}\left\{-p \cdot\left(F_{0}(X, a) \eta_{0}+\sum_{k=1}^{m} F_{k}(X, a) \eta_{k}\right)\right\} .
$$

The following result gives the characterization of $w$.

TheOREM 5.5. Assume that (H1) holds. Then $w$ is locally Lipschitz continuous and it is the unique viscosity solution to

$$
\begin{cases}\min \left\{\partial_{s} w(s, X)+H(X, D w(s, X)), w(s, X)-g(X)\right\}=0 & \text { in }(0,+\infty) \times \mathbb{R}^{n+m}, \\ w(0, X)=\max \{\varphi(X), g(X)\} & \text { in } \mathbb{R}^{n+m} .\end{cases}
$$

Before giving the proof for Theorem 5.5, we need the following dynamical programming principle (DPP) for $w$.

Proposition 5.6 (DPP). Assume (H1). For all $s \geq 0, X \in \mathbb{R}^{n+m}, h \geq 0, w$ satisfies

$$
w(s, X)=\inf \left\{\max \left(w(s-h, Z(h)), \max _{\theta \in[0, h]} g(Z(\theta))\right): Z \text { satisfies }(3.3)\right\} .
$$

The first consequence of the DPP is the local Lipschitz continuity of $w$.

Proposition 5.7. If (H1) holds, then $w$ is locally Lipschitz continuous.

Proof. For any $s \geq 0$, we start by proving the Lipschitz continuity of $w(s, \cdot)$. Let $X_{1}, X_{2} \in \mathbb{R}^{n+m}$, and denote for any $\alpha \in \mathcal{A}, \gamma \in \Gamma$, by $Z_{X_{i}}^{\alpha, \gamma}$ the solution to (3.3) with the control $\alpha, \gamma$ and the initial position $X_{i}$ for $i=1,2$. By the Lipschitz continuity of the dynamics and Gronwall's lemma, we have

$$
\left|Z_{X_{1}}^{\alpha, \gamma}(s)-Z_{X_{2}}^{\alpha, \gamma}(s)\right| \leq e^{L s}\left|X_{1}-X_{2}\right| .
$$

By the definition of $w$ and the inequalities

$$
\begin{gathered}
\inf A_{\alpha}-\inf B_{\alpha} \leq \sup \left(A_{\alpha}-B_{\alpha}\right), \\
\max \{A, B\}-\max \{C, D\} \leq \max \{A-C, B-D\},
\end{gathered}
$$

we obtain

$$
\begin{aligned}
& \left|w\left(s, X_{1}\right)-w\left(s, X_{2}\right)\right| \\
\leq & \sup _{\alpha \in \mathcal{A}, \gamma \in \Gamma} \max \left(\left|\varphi\left(Z_{X_{1}}^{\alpha, \gamma}(s)\right)-Z_{X_{2}}^{\alpha, \gamma}(s)\right|, \max _{\theta \in[0, s]}\left|g\left(Z_{X_{1}}^{\alpha, \gamma}(\theta)\right)-g\left(Z_{X_{2}}^{\alpha, \gamma}(\theta)\right)\right|\right) \\
\leq & \max \left\{L_{\varphi}, L_{g}\right\} e^{L s}\left|X_{1}-X_{2}\right|,
\end{aligned}
$$


where $L_{\varphi}$ and $L_{g}$ are the Lipschitz constants of $\varphi$ and $g$ respectively.

Now for any $X \in \mathbb{R}^{n+m}$, we proceed to show the local Lipschitz continuity of $w(\cdot, X)$. Let $s_{1}, s_{2} \geq 0$, assume without loss of generality that $s_{1} \geq s_{2}$. By the DPP, we obtain

$$
\begin{aligned}
& \left|w\left(s_{1}, X\right)-w\left(s_{2}, X\right)\right| \\
= & \left|\inf _{\alpha \in \mathcal{A}, \gamma \in \Gamma} \max \left(w\left(s_{2}, Z\left(s_{1}-s_{2}\right)\right), \max _{\theta \in\left[0, s_{1}-s_{2}\right]} g(Z(\theta))\right)-\max \left(w\left(s_{2}, X\right), g(X)\right)\right| \\
\leq & \sup _{\alpha \in \mathcal{A}, \gamma \in \Gamma} \max \left(\left|w\left(s_{2}, Z\left(s_{1}-s_{2}\right)\right)-w\left(s_{2}, X\right)\right|, \max _{\theta \in\left[0, s_{1}-s_{2}\right]}|g(Z(\theta))-g(X)|\right) \\
\leq & \max \left\{\max \left\{L_{\varphi}, L_{g}\right\} e^{L s_{2}}, L_{g}\right\}\left(M+m M C_{1}\right)\left|s_{1}-s_{2}\right|,
\end{aligned}
$$

which completes the proof. $\square$ Now we recall the definition of viscosity solution to (5.4).

Definition 5.8. Let $u:[0,+\infty) \times \mathbb{R}^{n+m} \rightarrow \mathbb{R}$.

- $u$ is a supersolution to (5.4) if $u$ is lower semi-continuous and for any $(s, X) \in$ $(0,+\infty) \times \mathbb{R}^{n+m}, \phi \in C^{1}\left((0,+\infty) \times \mathbb{R}^{n+m}\right)$ such that $u-\phi$ attains a local minimum at $(s, X)$, it holds that

$$
\min \left\{\partial_{s} \phi(s, X)+H(X, D \phi(s, X)), u(s, X)-g(X)\right\} \geq 0 .
$$

- $u$ is a subsolution to (5.4) if $u$ is upper semi-continuous and for any $(s, X) \in$ $(0,+\infty) \times \mathbb{R}^{n+m}, \phi \in C^{1}\left((0,+\infty) \times \mathbb{R}^{n+m}\right)$ such that $u-\phi$ attains a local maximum at $(s, X)$, it holds that

$$
\min \left\{\partial_{s} \phi(s, X)+H(X, D \phi(s, X)), u(s, X)-g(X)\right\} \leq 0 .
$$

- $u$ is a viscosity solution to (5.4) if $u$ is both a supersolution and a subsolution and $u$ satisfies the initial conditions

$$
u(0, X)=\max \{\varphi(X), g(X)\} .
$$

We now give the proof of Theorem 5.5.

Proof. At first, we will check the supersolution property of $w$. For any $(s, X) \in$ $(0,+\infty) \times \mathbb{R}^{n+m}$, by the DPP

$$
w(s, X) \geq \inf _{\alpha \in \mathcal{A}, \gamma \in \Gamma} w(s-h, Z(h)), \forall h \geq 0,
$$

and

$$
w(s, X) \geq \max _{\theta \in[0, h]} g(Z(\theta)) \geq g(X) .
$$

For each $\varepsilon>0$, there exists $Z^{\varepsilon}$ with the associated control $\left(\alpha^{\varepsilon}, \gamma^{\varepsilon}\right)$ such that

$$
w(s, X) \geq w\left(s-h, Z^{\varepsilon}(h)\right)-h \varepsilon, \forall h \geq 0 .
$$

Then for any $\phi \in C^{1}\left((0,+\infty) \times \mathbb{R}^{n+m}\right)$ such that $w-\phi$ attains a local minimum at $(s, X)$, we have

$$
\phi(s, X)-\phi\left(s-h, Z^{\varepsilon}(h)\right) \geq-h \varepsilon, \forall h \geq 0 .
$$


Thus,

$$
\frac{1}{h} \int_{0}^{h}\left[\partial_{s} \phi\left(s-h+\tau, Z^{\varepsilon}(\tau)\right)-D \phi\left(s-h+\tau, Z^{\varepsilon}(h)\right) \cdot \dot{Z}^{\varepsilon}(\tau)\right] d \tau \geq-\varepsilon, \forall h>0,
$$

i.e. $\forall h>0$

$$
\begin{aligned}
& \frac{1}{h} \int_{0}^{h}\left[\partial_{s} \phi\left(s-h+\tau, Z^{\varepsilon}(\tau)\right)-D \phi\left(s-h+\tau, Z^{\varepsilon}(h)\right) \cdot \sum_{k=0}^{m} F_{k}\left(Z^{\varepsilon}(\tau), \alpha^{\varepsilon}(\tau)\right) \dot{\gamma}_{k}^{\varepsilon}(\tau)\right] d \tau \\
& \geq-\varepsilon
\end{aligned}
$$

Using the fact that $\alpha^{\varepsilon}(\tau) \in A$ where $A$ is compact, we deduce by letting $h \rightarrow 0^{+}$that

$$
\partial_{s} \phi(s, X)-D \phi(s, X) \cdot \sum_{k=0}^{m} F_{k}\left(X, a^{\varepsilon}\right) \eta_{k}^{\varepsilon} \geq-\varepsilon,
$$

where $a^{\varepsilon} \in A$ and $\left(\eta_{0}^{\varepsilon}, \ldots, \eta_{m}^{\varepsilon}\right) \in c o \Theta$. The above inequality implies that

$$
\left.\partial_{s} \phi(s, X)+\sup _{a \in A, \eta \in c o}\{-D \phi(s, X)) \cdot \sum_{k=0}^{m} F_{k}(X, a) \eta_{k}\right\} \geq-\varepsilon, \forall \varepsilon>0,
$$

which is equivalent to

$$
\partial_{s} \phi(s, X)+H(X, D \phi(s, X)) \geq-\varepsilon, \forall \varepsilon>0 .
$$

The arbitrary choice of $\varepsilon$ implies that

$$
\partial_{s} \phi(s, X)+H(X, D \phi(s, X)) \geq 0,
$$

which concludes the supersolution property.

We proceed to check the subsolution property of $w$. For any $(s, X) \in(0,+\infty) \times$ $\mathbb{R}^{n+m}$, if $w(s, X) \leq g(X)$, then the property is trivial to prove. Otherwise, there exists some small enough $h>0$ such that for all $Z(\cdot)$ satisfying $(3.3)$

$$
w(s, X)>\max _{\theta \in[0, h]} g(Z(\theta)) .
$$

Then by the DPP,

$$
w(s, X) \leq \max \left(w(s-h, Z(h)), \max _{\theta \in[0, h]} g(Z(\theta))\right),
$$

for all all $Z(\cdot)$ satisfying (3.3). Together with (5.5), it follows that

$$
w(s, X) \leq w(s-h, Z(h)) .
$$

For each $a \in A, \eta \in \Theta$, we set $\alpha(\cdot) \equiv a$. The aim is to find $u \in \mathcal{U}$ and $\gamma \in \Theta$ such that

$$
\lim _{h \rightarrow 0^{+}} \frac{\gamma(h)-\gamma(0)}{h}=\eta .
$$

Consider the following two cases. 
Case 1: $\eta_{0}=0$. In this case, $0 \in \mathcal{T}$, then we can take any $u \in \mathcal{U}$ with

$$
u^{k}\left(0^{+}\right)-u^{k}\left(0^{-}\right)=\frac{\eta_{k}}{\sum_{i=1}^{m}\left|\eta_{i}\right|}=\eta_{k}, \forall k=1, \ldots, m .
$$

Thus, $\dot{\gamma}_{k}\left(0^{+}\right)=\eta_{k}$ for all $k=0, \ldots, m$.

Case 2: $\eta_{0}>0$. In this case, no jump occurs at time 0 . We then take any $u \in \mathcal{U}$ with

$$
\dot{u}^{k}\left(0^{+}\right)=\frac{\eta_{k}}{\eta_{0}}, \forall k=1, \ldots, m,
$$

which ensures $\dot{\gamma}_{k}\left(0^{+}\right)=\eta_{k}$ for all $k=0, \ldots, m$.

Now let $Z^{\alpha, \gamma}$ be the solution to (3.3) with the controls $\alpha, \gamma$ constructed as above. For any $\phi \in C^{1}\left((0,+\infty) \times \mathbb{R}^{n+m}\right)$ such that $w-\phi$ attains a local maximum at $(s, X)$, together with (5.6) we have

$$
\phi(s, X) \leq \phi\left(s-h, Z^{\alpha, \gamma}(h)\right), \forall h \geq 0,
$$

i.e. $\forall h>0$

$$
\begin{aligned}
& \frac{1}{h} \int_{0}^{h}\left[\partial_{s} \phi\left(s-h+\tau, Z^{\alpha, \gamma}(\tau)\right)\right. \\
& \left.-D \phi\left(s-h+\tau, Z^{\alpha, \gamma}(h)\right) \cdot \sum_{k=0}^{m} F_{k}\left(Z^{\alpha, \gamma}(\tau), a\right) \dot{\gamma}^{k}(\tau)\right] d \tau \leq 0 .
\end{aligned}
$$

Up to a subsequence, it holds that $\dot{\gamma}^{k}\left(h_{n}\right) \rightarrow \eta_{k}$ for any $k=0, \ldots, m$. Then we have

$$
\partial_{s} \phi(s, X)-D \phi(s, X) \cdot \sum_{k=0}^{m} F_{k}(x, a) \eta_{k} \leq 0,
$$

for all $a \in A, \eta \in \Theta$. Hence,

$$
\partial_{s} \phi(s, X)+H(X, D w(s, X)) \leq 0,
$$

which concludes the subsolution property.

The definition of $w$ implies that $w$ satisfies the initial condition. The uniqueness of $v$ is a direct result of the following comparison principle. This completes the proof. (

Theorem 5.9 (Comparison principle). Assume (H1). Let $w_{1}, w_{2}:[0, \infty) \times$ $\mathbb{R}^{n+m}$ be respectively a subsolution and a supersolution to (5.5) and $w_{1}(0, X) \leq$ $w_{2}(0, X)$ for all $X \in \mathbb{R}^{n+m}$. Then

$$
w_{1} \leq w_{2} \text { in }[0, \infty) \times \mathbb{R}^{n+m} .
$$

Proof. The definition of supersolution gives that

$$
\partial_{s} \phi(s, X)+H\left(X, D w_{2}(s, X)\right) \geq 0 \text { and } w_{2}(s, X) \geq g(X) \text { in }[0, \infty) \times \mathbb{R}^{n+m} .
$$

The definition of subsolution leads to

$$
\partial_{s} \phi(s, X)+H\left(X, D w_{1}(s, X)\right) \leq 0 \text { or } w_{1}(s, X) \leq g(X) \text { in }[0, \infty) \times \mathbb{R}^{n+m} .
$$

If $w_{1}(s, X) \leq g(X)$, then $w_{1}(s, X) \leq g(X) \leq w_{2}(s, X)$. Otherwise, we conclude that $w_{1} \leq w_{2}$ by $[3$, Theorem III.3.7]. 
6 Applications We consider the following time optimal control of a riderand-swing system discussed in [17]. This system is modeled as a pendulum with a massless rope. The state variable is the angle $\theta$ of the pendulum with the vertical, and the control variable is the length of the pendulum $\ell$. Then the state equation is

$$
\ddot{\theta}(t)+\frac{2 \dot{\ell}(t) \dot{\theta}(t)}{\ell(t)}+\frac{g \sin (\theta(t))}{\ell(t)}=0, \text { for } t \in(0, \infty),
$$

where $\ell(t) \in\left[L^{-}, L^{+}\right]$. Given a target $\mathcal{C}$ as a closed subset of $\mathbb{R}$, the minimal time problem is the following:

$$
T\left(\theta_{0}, \ell_{0}\right):=\inf \left\{t: \theta(t) \in \mathcal{C},(\theta, \ell) \text { satisfies }(6.1),(\theta, \ell)(0)=\left(\theta_{0}, \ell_{0}\right)\right\} .
$$

By introducing $Y=\left(y_{1}, y_{2}, y_{3}\right)=(\theta, \dot{\theta}, \ell)$, the equation (6.1) is reformulated as

$$
\left\{\begin{array}{l}
\dot{y}_{1}=y_{2} \\
\dot{y}_{2}=-\frac{g \sin \left(y_{1}\right)}{y_{3}}-\frac{2 y_{2}}{y_{3}} \dot{\ell} \\
\dot{y}_{3}=\dot{\ell} .
\end{array}\right.
$$

Then the reparametrized system is the following

$$
\left\{\begin{array}{l}
\dot{z}_{1}(s)=z_{2}(s) \dot{\gamma}_{0}(s) \\
\dot{z}_{2}(s)=-\frac{g \sin \left(z_{1}(s)\right)}{z_{3}(s)} \dot{\gamma}_{0}(s)-\frac{2 z_{2}(s)}{z_{3}(s)} \dot{\gamma}_{1}(s) \\
\dot{z}_{3}(s)=\dot{\gamma}_{1}(s),
\end{array}\right.
$$

where $\left(\gamma_{0}, \gamma_{1}\right)$ is the canonical graph completion of $\ell$. As defined in (4.1), for any $X \in \mathbb{R}^{3}$ the reparametrized control problem is

$$
S(X):=\inf \left\{s \geq 0: Z(0)=X, z_{1}(s) \in \mathcal{C}, z_{3}(\tau) \in\left[L^{-}, L^{+}\right], \forall \tau \in[0, s]\right\}
$$

Let $\varphi, g: \mathbb{R}^{3} \rightarrow \mathbb{R}$ be the signed distance function to $\mathcal{C} \times \mathbb{R}^{2}$ and $\mathbb{R}^{2} \times\left[L^{-}, L^{+}\right]$ respectively. Then $w$ is defined as

$$
w(s, X):=\inf \left\{\max \left(\varphi(Z(s)), \max _{\theta \in[0, s]} g(Z(\theta))\right): Z \text { satisfies }(6.3)\right\} .
$$

We solve the following HJB equation to obtain the 0 -sublevel set of $w$ :

$$
\begin{cases}\min \left\{\partial_{s} w(s, X)+H(X, D w(s, X)), w(s, X)-g(X)\right\}=0 & \text { for } s \in(0,+\infty), X \in \mathbb{R}^{3}, \\ w(0, X)=\max \{\varphi(X), g(X)\} & \text { for } X \in \mathbb{R}^{3},\end{cases}
$$

where the Hamiltonian $H: \mathbb{R}^{3} \times \mathbb{R}^{3} \rightarrow \mathbb{R}$ is defined by

$$
H(X, p):=\sup _{\eta=\left(\eta_{0}, \eta_{1}\right) \in \Theta}\left\{-p \cdot\left(x_{2} \eta_{0},-\frac{g \sin \left(x_{1}\right)}{x_{3}} \eta_{0}-\frac{2 x_{2}}{x_{3}} \eta_{1}, \eta_{1}\right)\right\} .
$$

In the first simulation, we take $L^{-}=9, L^{+}=11$ and the target set

$$
\mathcal{C} \times \mathbb{R}^{2}=\left\{\left(x_{1}, x_{2}, x_{3}\right): x_{1} \geq \pi / 3\right\} .
$$

Let $\varphi$ and $g$ be the following:

$\varphi(x)=\min \left\{\frac{\pi}{3}-x_{1}, 0.4\right\}, g(x)=\max \left\{9-x_{3}, x_{3}-11\right\}$ for any $x=\left(x_{1}, x_{2}, x_{3}\right) \in \mathbb{R}^{3}$. 


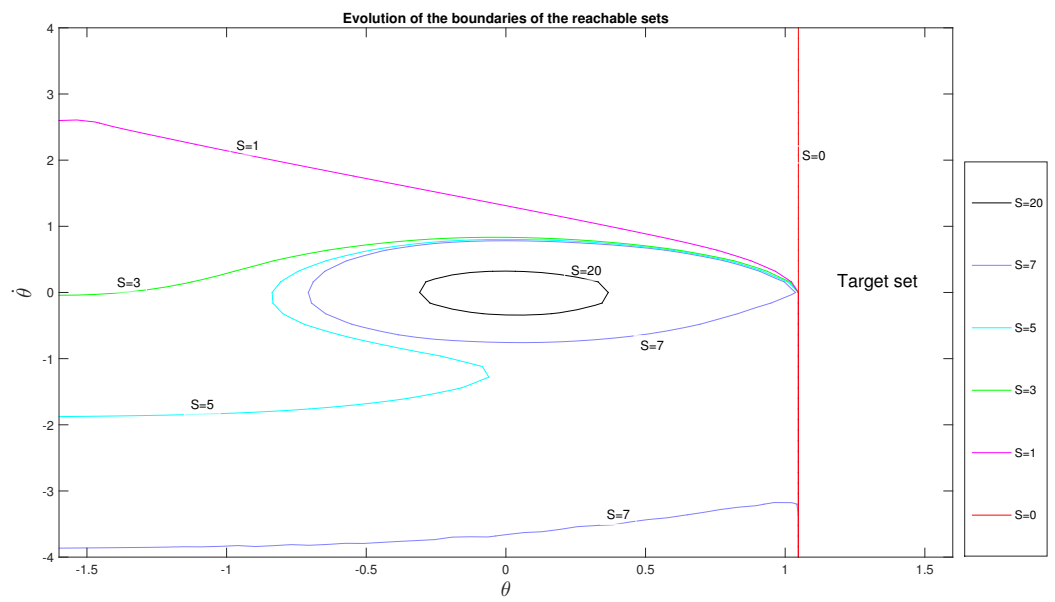

Fig. 6.1. Evolution of the boundaries of the capture basin for the target set.

The computing domain is $[-1.6,1.6] \times[-4,4] \times[8,12]$ with $50^{3}$ mesh points. The numerical test uses the software ROC-HJ solver developed by Bokanowski, Desilles and Zidani (http://itn-sadco.inria.fr/software/ROC-HJ). Figure 6.1 shows the evolution of the capture basin for the target $\mathcal{C}$ on the surface $\left\{\left(x_{1}, x_{2}, x_{3}\right) \in \mathbb{R}^{3}: x_{3}=10\right\}$, and figure 6.2 illustrates the graphs of $w$ at different time. We remark that after a long time, the complement set of the capture basin becomes a neighborhood of the origin $(0,0)$ which can not be reached in finite time.
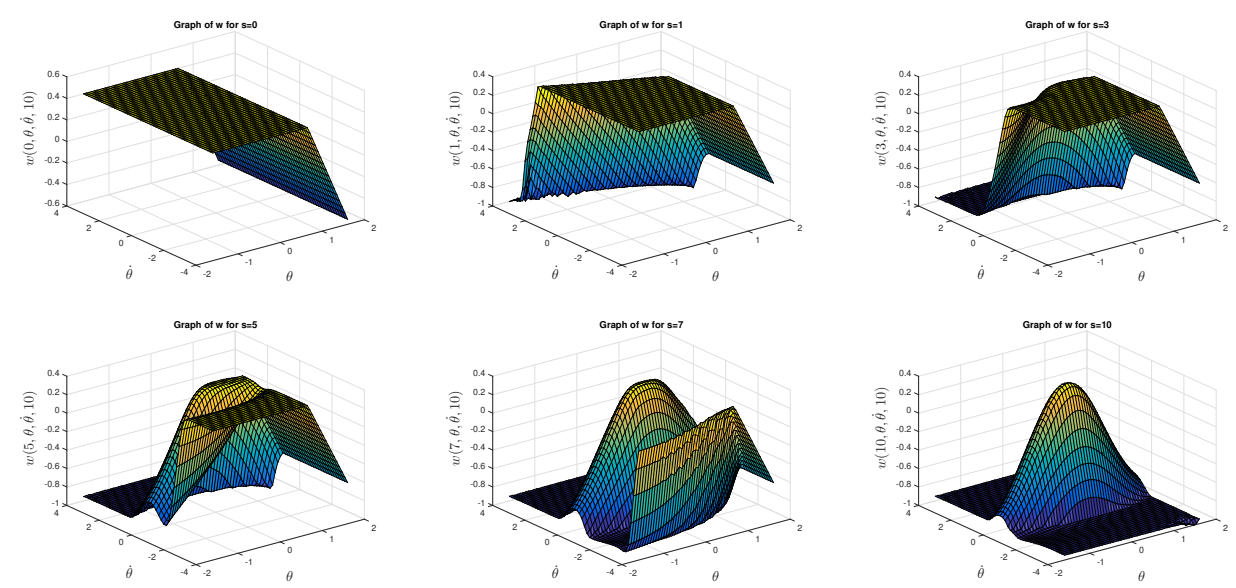

FIG. 6.2. Graph of $w(s, \theta, \dot{\theta}, 10)$ at time $s=0,1,3,5,7,10$.

Finally, we give a comparison between the above problem with the impulsive control $\dot{\ell}$ and the problem without this type of control, i.e. consider the following state equation

$$
\ddot{\theta}(t)+\frac{g \sin (\theta(t))}{\ell(t)}=0, \text { for } t \in(0, \infty) .
$$


Figure 6.3 gives the boundaries of the capture basin in both cases at the same final time $S=10$. We remark that the unreachable set in the case with impulsive controls is smaller than the one without impulsive controls.

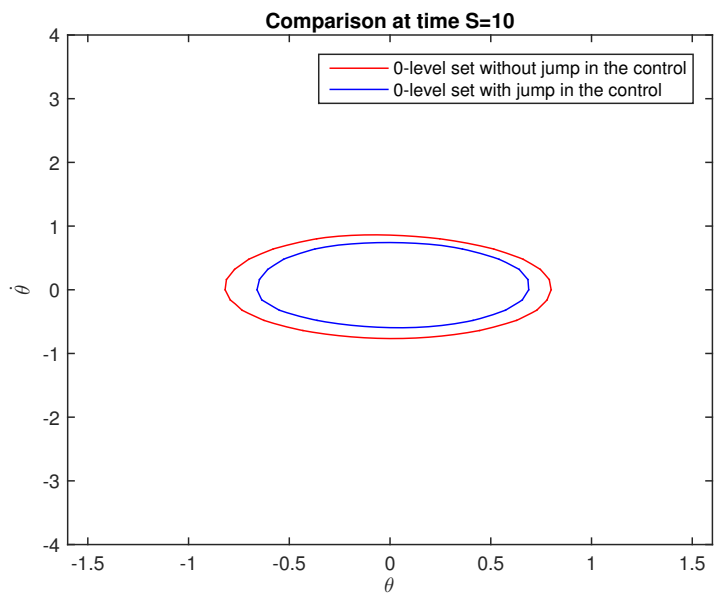

FIG. 6.3. Reachable sets with/without impulsive controls.

\section{REFERENCES}

[1] L. Ambrosio, N. Fusco and D. Pallara, Functions of bounded variation and free discontinuity problems, Oxford Mathematical Monographs, The Clarendon Press, Oxford University Press, New York, 2000.

[2] S. Aronna and F. Rampazzo, On optimal control problems with impulsive commutative dynamics, Proceedings of the 52nd IEEE Conference on Decision and Control 2013, 1822-1827.

[3] M. Bardi and I. Capuzzo Dolcetta, Optimal control and viscosity solutions of Hamilton-JacobiBellman equations, Systems and Control: Foundations and Applications, Birkhäuser, Boston, 1997.

[4] A. Bensoussan and J.L. Lions, Impulse control and quasi-variational inequalities, GauthierVillars, 1984.

[5] O. Bokanowski, N. Forcadel and H. Zidani, Reachability and minimal times for state constrained nonlinear problems without any controllability assumption, SIAM J. Control and Optimization., 48(7) (2010), 4292-4316.

[6] A.Bressan and F. Rampazzo, On differential systems with vector-valued impulsive controls, Boll. Un. Mat. Ital. 7(2-B) (1988), 641-656.

[7] A. Bressan and F. Rampazzo, Impulsive control-systems with commutativity assumptions, J. Optim. Theory Appl., 71(1) (1991), 67-83.

[8] A. Bressan and F. Rampazzo, Impulsive control-systems without commutativity assumptions, J. Optim. Theory Appl., 81(3) (1994), 435-457.

[9] A. Briani and H. Zidani, Characterization of the value function of final state constrained control problems with BV trajectories, Communication in Pure and Applied Analysis, vol. 10(6) (2011), 1567-1587.

[10] A. Catlla, D. Schaeffer, T. Witelski, E. Monson and A. Lin, On spiking models for synaptic activity and impulsive differential equations, SIAM Rev. 50(3) (2005), 553-569.

[11] F.H. Clarke, Yu.S. Ledyaev, R.J. Stern and P.R. Wolenski, Nonsmooth Analysis and Control Theory, Graduate Texts in Mathematics 178, Springer-Verlag, New York.

[12] G. Dal Maso and F. Rampazzo, On systems of ordinary differential equations with measures as controls, Differential and Integral Equations, 4 (1991), 739-765.

[13] P. Gajardo, C. Ramirez and C. Rapaport, Minimal time sequential batch reactors with bounded and impulse controls for one or more species, SIAM J. Control Optim., 47(6) (2008), 28272856. 
[14] N. Forcadel, Z. Rao and H. Zidani, State constrained optimal control problems of impulsive differential equations, Appl. Math. Optim., 68 (2013), 1-19.

[15] M. Motta and F. Rampazzo, Dynamic programming for nonlinear systems driven by ordinary and impulsive controls, SIAM J. Control Optim. 34(1) (1996), 199-225.

[16] F.L. Pereira and G.N. Silva, Necessary conditions of optimality for vector-valued impulsive control problems, Systems Control Lett., 40(3) (2000), 205-215.

[17] B. Piccoli, Time-optimal control problems for the swing and the ski, International Journal of Control 62(6) (1995), 1409-1429.

[18] Z. Rao, Hamilton-Jacobi-Bellman approach for optimal control problems with discontinuous coefficients, https://pastel.archives-ouvertes.fr/pastel-00927358.

[19] R.W. Rishel, An extended Pontryagin principle for control systems whose control laws contain measures, SIAM J. Control 3(2) (1965), 191-205.

[20] G.N. Silva and R.B. Vinter, Measure driven differential inclusions, J. of Math. Anal. and Appl. 202 (1996), 746-767.

[21] G.N. Silva and R.B. Vinter, Necessary conditions for optimal impulsive control problems, SIAM J. Control Optim. 35(6) (1998), 1829-1846.

[22] P.R. Wolenski and S. Zabic, A differential solution concept for impulsive systems, Differential Equations and Dynamical Systems, 2 (2006), 199-210. 